\title{
On the 3-D Shape of Interlaced Regions in Sn-3Ag-0.5Cu Solder Balls
}

\author{
A.A. DASZKI (1D ${ }^{1,2}$ and C.M. GOURLAY ${ }^{1}$ \\ 1.-Department of Materials, Imperial College London, London SW7 2AZ, UK. 2.-e-mail: \\ armin.daszki12@imperial.ac.uk
}

The microstructure of $\mathrm{Sn}-\mathrm{Ag}-\mathrm{Cu}$ (SAC) solder joints plays an important role in the reliability of electronics, and interlaced twinning has been linked with improved performance. Here, we study the three-dimensional (3-D) shape of interlaced regions in Sn-3.0Ag-0.5Cu (SAC305) solder balls by combining serial sectioning with electron backscatter diffraction. In solder balls without large $\mathrm{Ag}_{3} \mathrm{Sn}$ plates, we show that the interlaced volume can be reasonably approximated as a hollow double cone with the common $\langle 100\rangle$ twinning axis as the cone axis, and the $\langle 110\rangle$ from all three twinned orientations making up the cone sides. This $3-\mathrm{D}$ morphology can explain a range of partially interlaced morphologies in past work on 2 -D cross-sections.

Key words: Lead-free solder, interlacing, microstructure, electron backscatter diffraction (EBSD), twinning

\section{INTRODUCTION}

$\beta \mathrm{Sn}$ is the main solid phase in most electronic solder joints, ${ }^{1}$ and dictates much of the electrical and mechanical properties of joints. The bodycentered tetragonal (bct) structure is highly anisotropic. For example, the coefficient of thermal expansion $^{2,3}$ and solute diffusivity ${ }^{4}$ vary between the $a$-direction and the $c$-direction of $\beta \mathrm{Sn}$. In leadfree solder joints, $\beta$ Sn usually presents only a few grain orientations, with single grain joints ${ }^{5-7}$ and three grain morphologies ${ }^{1,5,8-11}$ frequently reported in $\mathrm{Sn}-\mathrm{Ag}-\mathrm{Cu}(\mathrm{SAC})$ solders. The three orientations of $\beta \mathrm{Sn}$ are related via cyclic twinning around a common $\langle 100\rangle$ direction, ${ }^{1,6}$ and seem to come from a single nucleation event. These cyclic twin microstructures have been reported as either a beach-ball or interlacing morphology, or a mixture of the two. ${ }^{9,12}$ Electron backscatter diffraction (EBSD) techniques have been successfully used to identify crystal orientations and relationships

(Received July 16, 2020; accepted September 21, 2020; published online October 15, 2020) between $\beta$ Sn grains, such as the common twinning axis. $1,7,13,14$

A SAC solder joint with an interlaced microstructure, composed of many small $\beta \mathrm{Sn}$ grains, shows increased mechanical properties, such as hardness and creep, ${ }^{15}$ as well as better reliability compared to a beach-ball microstructure, by delaying crack initiation. ${ }^{16}$ Furthermore, a solder joint of a single grain will have anisotropic mechanical properties, corresponding to $\beta \mathrm{Sn}$ anisotropy ${ }_{17}^{17}$ and can result in early joint failure for certain grain orientations. The ability to control or predict interlacing in solder joints could therefore be beneficial in improving their reliability.

Lehman et al. ${ }^{1}$ proposed that the beach-ball morphology comes from $\{101\}$ cyclic twinning, and that the interlaced morphology comes from \{301\} cyclic twinning. However, many SAC microstructures have an interlaced morphology near the apparent nucleation point and a beach ball morphology growing away from the interlaced region. 8,11 Arfaei et al. proposed that the difference between the interlaced and beach-ball morphologies may be due to a change in preferred growth direction of $\beta \mathrm{Sn}$ dendrites, from $\langle 110\rangle\rangle^{8}$ Their research into the interlaced nature of $\beta \mathrm{Sn}$ grains 
in SAC solder balls and joints concluded that increased $\beta \mathrm{Sn}$ nucleation undercooling (lower solidification temperature) favours larger areas of interlacing. $^{8}$

Past research of $\mathrm{Sn}-\mathrm{Ag}-\mathrm{Cu}$ solder balls contain micrographs of partially-interlaced regions with a range of morphologies. Examination of past papers revealed two frequently observed microstructures: a crescent shape and a ring of interlacing surrounded by a $\beta \mathrm{Sn}$ dendritic region,,${ }^{1,9,18-20}$ as listed in Table I. These two microstructures share the common feature of a band of $\beta \mathrm{Sn}$ interlacing between beach-ball-type cyclic twinned dendrites. While these two microstructural features are shown in various different papers, past work has not considered them in detail, and it is not clear if these shapes in two-dimensional (2-D) cross-sections belong to the same shape in three-dimensions (3D). From micrographs in the literature, it also seems that these microstructures tend to form when there is a lack of primary IMC particles.

This study aims to build a deeper understanding of the formation of interlaced twinning in SAC solders by exploring the crescent and ring microstructures of $\beta \mathrm{Sn}$ interlacing in $\mathrm{Sn}-3.0 \mathrm{Ag}-$ $0.5 \mathrm{Cu}$ (SAC305) solder balls. We focus on reconstructing the 3-D shape of interlaced regions by combining serial sectioning and EBSD.

\section{METHODS}

The majority of this study was performed on a SAC305 solder alloy made by mixing high-purity $\mathrm{Sn}, \mathrm{Ag}$ and a $\mathrm{Sn}-10 \mathrm{Cu}$ master alloy and casting into an ingot. The composition of the alloy (wt\%) was measured by XRF spectroscopy to be Sn-3.2Ag$0.47 \mathrm{Cu}$, with main impurities of $0.018 \mathrm{Sb}, 0.006 \mathrm{~Pb}$ and $0.002 \mathrm{Ni}$, and $\mathrm{Co}, \mathrm{Fe}, \mathrm{Zn}$ and $\mathrm{Bi}$, all $<0.001 \mathrm{wt} \%$. To test for the generality of the findings, we also studied a small number of samples made from two further batches of SAC305: one from commercial ingot supplied by Nihon Superior, Osaka, Japan, and another made from commercial purity starting materials from our past work in Ref. 7.

The cast ingots were then cut into small pieces which were rolled into a foil of approximately $50 \mu \mathrm{m}$ thickness. Small 1.5-mm-diameter disks were punched out of the foils and then melted in a ROL1 (IPC J-STD-004) (Nihon Superior) flux on a hotplate at $240^{\circ} \mathrm{C}$. The disks melted and formed spheres of approximately $500 \mu \mathrm{m}$ diameter under surface tension. The solder balls were then cleaned in an ultrasonic bath, with ethanol as a cleaning solvent.

Solder balls were then placed in an aluminium pan and run in a Mettler Toledo differential scanning calorimeter to melt and solidify them, and to measure the solidification temperature. All the samples, unless stated, were heated up to a peak of $240^{\circ} \mathrm{C}$ and cooled with a cooling rate of $-20 \mathrm{~K} \mathrm{~min}^{-1}\left(-0.333 \mathrm{~K} \mathrm{~s}^{-1}\right)$ under a $\mathrm{N}_{2}$ gas atmosphere. Some samples were cycled only once, and others were cycled multiple times, up to 12 times. One sample was heated to a peak temperature of $300^{\circ} \mathrm{C}$.

The samples were mounted in VersoCit coldsetting acrylic resin (Struers), ground on $\mathrm{SiC}$ grinding paper with water as lubricant, up to \#4000 grit, and polished with colloidal silica (OP-S; Struers). Micrographs of the samples were then taken under reflected light and polarised light microscopy. Some of the samples were ground in two orthogonal directions using serial sectioning to reveal the 3-D structure. These samples were ground and polished a few times, as normal, but not up to the apparent mid-section of the sample. They were then cut out of the resin and remounted at approximately right angles, and ground again, with multiple crosssection micrographs being taken throughout the rest of the sample. This is illustrated in Fig. 1.

Reconstruction of the sample volume was carried out in Inkscape image vector graphics software (Inkscape v.0.91). Figure 2 shows the method followed from Ref. 21 in creating the front projection and the top projection to create an isometric volume reconstruction of solder balls. The front projection was taken as the first two sections of the sample, and the top projection was taken as the crosssectioning after sample remounting. Aligning the samples in 3-D was approximated by lining up the chord length of the sections with the cross-section outline. This means that the presented volume reconstructions are not dimensionally exact, but serve well as an approximate representation of the sample volume. The micrographs were cropped and

Table 1. Observed crescent and ring morphologies of $\beta \mathrm{Sn}$ interlacing in the literature; this is not an exhaustive list of literature

\begin{tabular}{|c|c|c|c|c|}
\hline Author & Figure in the reference & Alloy & Morphology & References \\
\hline Lehman et al. & Figure 3c & $\mathrm{Sn}-3.9 \mathrm{Ag}-0.6 \mathrm{Cu}$ & Crescent & 1 \\
\hline \multirow{2}{*}{ Lehman et al. } & Figure $7 \mathrm{a}$ & $\mathrm{Sn}-3.0 \mathrm{Ag}$ & Crescent & 9 \\
\hline & Figure $7 \mathrm{~b}$ & $\mathrm{Sn}-3.0 \mathrm{Ag}-0.27 \mathrm{Cu}$ & Crescent & \\
\hline Mueller et al. & Figure 3 & $\mathrm{Sn}-3.0 \mathrm{Ag}-0.5 \mathrm{Cu}$ & Crescent & 18 \\
\hline \multirow[t]{2}{*}{ Panchenko et al. } & Figure 3 & $\mathrm{Sn}-3.0 \mathrm{Ag}-0.7 \mathrm{Cu}$ & Ring & 19 \\
\hline & Figure 6 & Various & Ring + crescent & \\
\hline Krause et al. & Figure 4 & Sn-3.0Ag-0.5Cu & Crescent & 20 \\
\hline
\end{tabular}




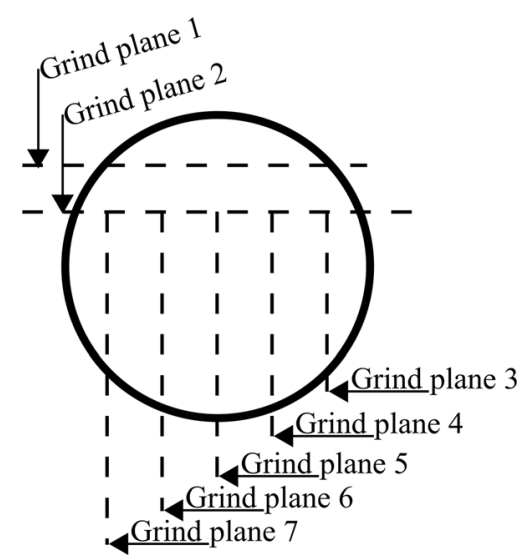

Fig. 1. Schematic of 3-D sample grinding. After a few sections, the samples were remounted at approximately right angles to create perpendicular cross-sections.

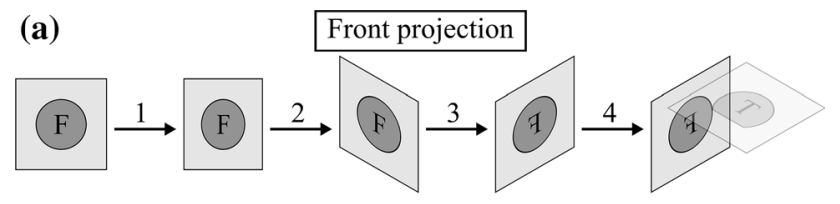

1. Scale width to $86.6 \%$

2. Skew vertically by $-30^{\circ}$

3. Flip horizontally to 'open' 4. Align

(b) Top projection

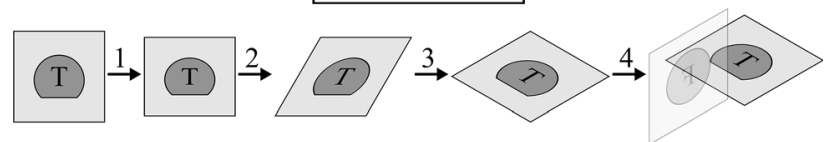

1. Scale height to $86.6 \%$

2. Skew horizontally by $-30^{\circ}$

3. Rotate by $30^{\circ}$ clockwise

4. Align

Fig. 2. The method followed in creating 3-D isometric projections of sample cross-sections. (a) Creating front projections; note that step 3 here flips the sample micrographs such that the observed microstructure is from within the page left-to-right; (b) creating top projections of the cross-sections.

contrast-corrected using ImageJ software (ImageJ $1.51 \mathrm{~h}){ }^{22}$

Samples were also analysed using EBSD (Bruker e-flash HR detector) in a scanning electron microscope (Zeiss Sigma FEG-SEM) to reveal crystal orientations in the samples. EBSD results were analysed in Bruker Esprit 2.1 software.

\section{RESULTS AND DISCUSSION}

Figure 3 shows the results of serial-sectioning, EBSD and reconstruction of a SAC305 solder ball with a melt undercooling of $47 \mathrm{~K}$. Figure $3 \mathrm{a}-\mathrm{f}$ illustrates how the interlacing shape changes through the volume of the sample and presents a crescent-shaped region of interlacing in Fig. 3b, d and e. Figure $3 \mathrm{i}$ shows the crescent interlacing region measured by EBSD, and the corresponding pole figure in Fig. 3j shows that the sample is made up of $3 \beta \mathrm{Sn}$ grain orientations, with a common $\{100\}$, whose normal direction is the twinning axis. From the 3-D reconstruction in Fig. 3m, we see that the interlaced area (red) starts as a circle in the lower right of the sample, then opens up into a ring and crescent shape towards the top right. Note that the front reconstruction of the microstructure has been flipped to open the 3-D structure, such that the microstructure observed in the microscope is reconstructed in the direction out of the page from left to right. EBSD results from all three cross-sections in Fig. $3 g-1$ show that only three $\beta$ Sn grain orientations were detected in this sample.

Figure 4 shows another example from a sample with a melt undercooling of $53 \mathrm{~K}$. The interlaced area has crescent, circular and ring-like shapes between sections. Again, from the reconstruction in Fig. $4 \mathrm{~m}$, the interlaced region can be seen to open up from the top right towards the bottom left, starting circular with a core-like feature in the centre, and creating a successively larger ring-like morphology. The crescent shape is observed as a perpendicular cross-section of the circular shape. The EBSD results shown in Fig. $4 \mathrm{~g}-1$ show that the sample only contains three $\beta \mathrm{Sn}$ grain orientations that are cyclic twinned, and we can clearly deduce that the perpendicular EBSD results show consistent grain orientations in the pole figures.

Microstructures in the two reconstructed samples are shown in more detail in Fig. 5. From the optical micrographs in Fig. $5 b$ and $f$, we see that the eutectic IMCs become finer towards the centre of the interlacing region. Likewise, the interlacing grain size decreases towards the centre of the region. This is consistent with past observations of these structures. ${ }^{18}$ From the polarised micrographs Fig. 5c and g, we see that the centre of these interlaced areas are actually made up of a few larger $\beta$ Sn grains which appear as a core surrounded by the interlacing region. This is confirmed by EBSD in Fig. 5d, with blue and purple grains substantially larger than the surrounding interlaced area. In further cross-sections, these cores increase in size and become dendritic $\beta \mathrm{Sn}$ regions inside the widening interlacing ring/crescent (Figs. 3, 4). This differs from the core structures reported by Mueller et al., ${ }^{18}$ which have a uniform circular cross-section and elongated ellipsoid shape.

From Figs. $3 \mathrm{~m}$ and $4 \mathrm{~m}$, the $3-\mathrm{D}$ shape of the envelope bounding the interlaced region can be deduced to be a hollow double cone. This proposed structure is explained in Fig. 6, with Fig. 6d showing the overall shape of this hollow double cone. Figure 6a shows how such a cone can be constructed with the [100] direction of $\beta$ Sn running along the cone axis, and the two $\langle 110\rangle$ directions inclined at $45^{\circ}$ to the axis. Figure $6 \mathrm{~b}$ shows how the commonly 


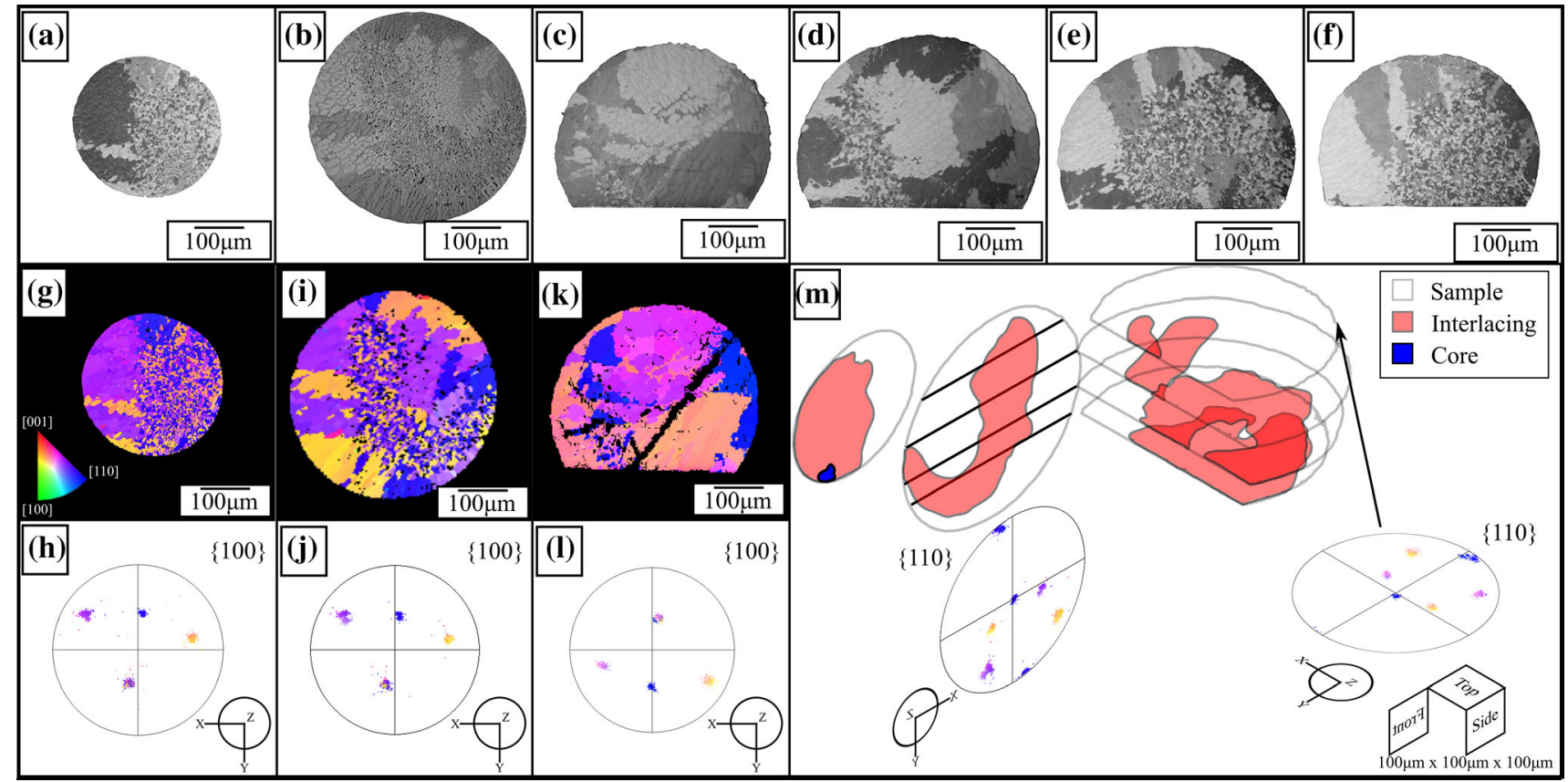

Fig. 3. 3-D cross-section reconstruction of SAC305 sample with an undercooling of $47 \mathrm{~K}$. (a-f) Polarised micrographs of cross-sectioned SAC305 solder ball; (g), (i), and (k) are IPFY EBSD maps of cross-sections from (a), (b) and (c), respectively, with the corresponding \{100\} pole figures shown in (h), (j) and (I), respectively; (m) 3-D isometric reconstruction of the sample with the interlacing and core areas in red and blue, respectively; $\{110\}$ pole figures in $(m)$ are from EBSD maps in (i) and $(k)$, and are transformed to closely align with the isometric projection. A $1 \times 10^{6} \mu \mathrm{m}^{3}$ cube is shown for scale in $(\mathrm{m})$, with the front face opened just like the reconstruction (Color figure online).

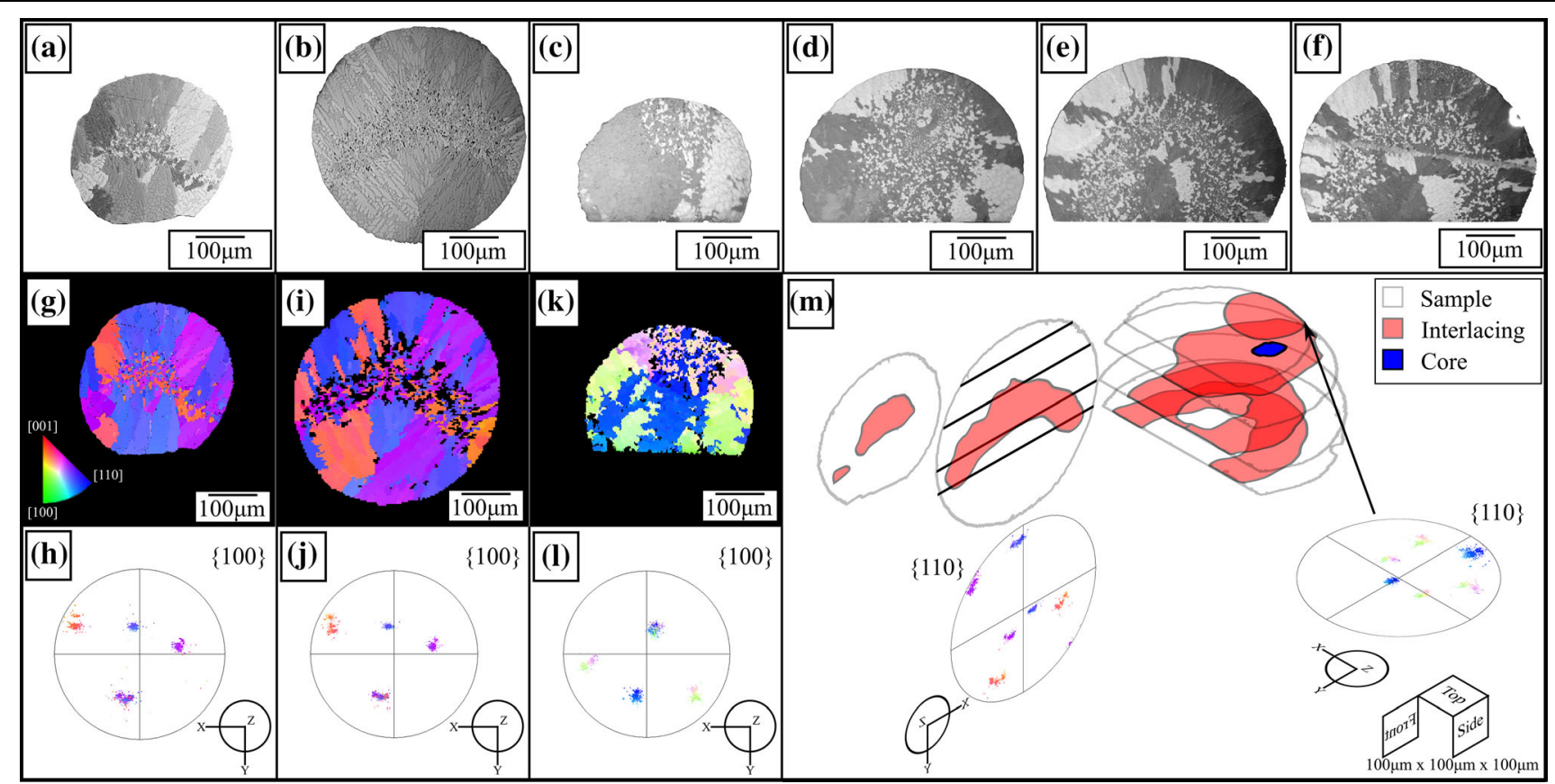

Fig. 4. 3-D cross-section reconstruction of SAC305 sample with an undercooling of $53 \mathrm{~K}$. (a-f) Polarised micrographs of cross-sectioned SAC305 solder ball; (g), (i), and (k) are IPFZ EBSD maps of cross-sections from (a), (b) and (c), respectively, with the corresponding \{100\} pole figures shown in (h), (j) and (I), respectively; (m) 3-D isometric reconstruction of the sample with the interlacing and core areas are red and blue, respectively; $\{110\}$ pole figures in $(\mathrm{m})$ are from EBSD maps in (i) and $(\mathrm{k})$, and are transformed to closely line up with the isometric projection. A $1 \times 10^{6} \mu \mathrm{m}^{3}$ cube is shown for scale in $(\mathrm{m})$, with the front face opened just like the reconstruction (Color figure online).

observed $\sim 60^{\circ}$ cyclic twinning around the shared [100] direction produces six $\langle 110\rangle$ directions revolved around the cone axis forming the cone sides. Powell et al. ${ }^{23}$ have shown that $\beta$ Sn growth often deviates from $\langle 110\rangle$, with a $c$-component to growth, i.e. $\langle 11 w\rangle$ with $0<w \leq 1$. In this case, there 


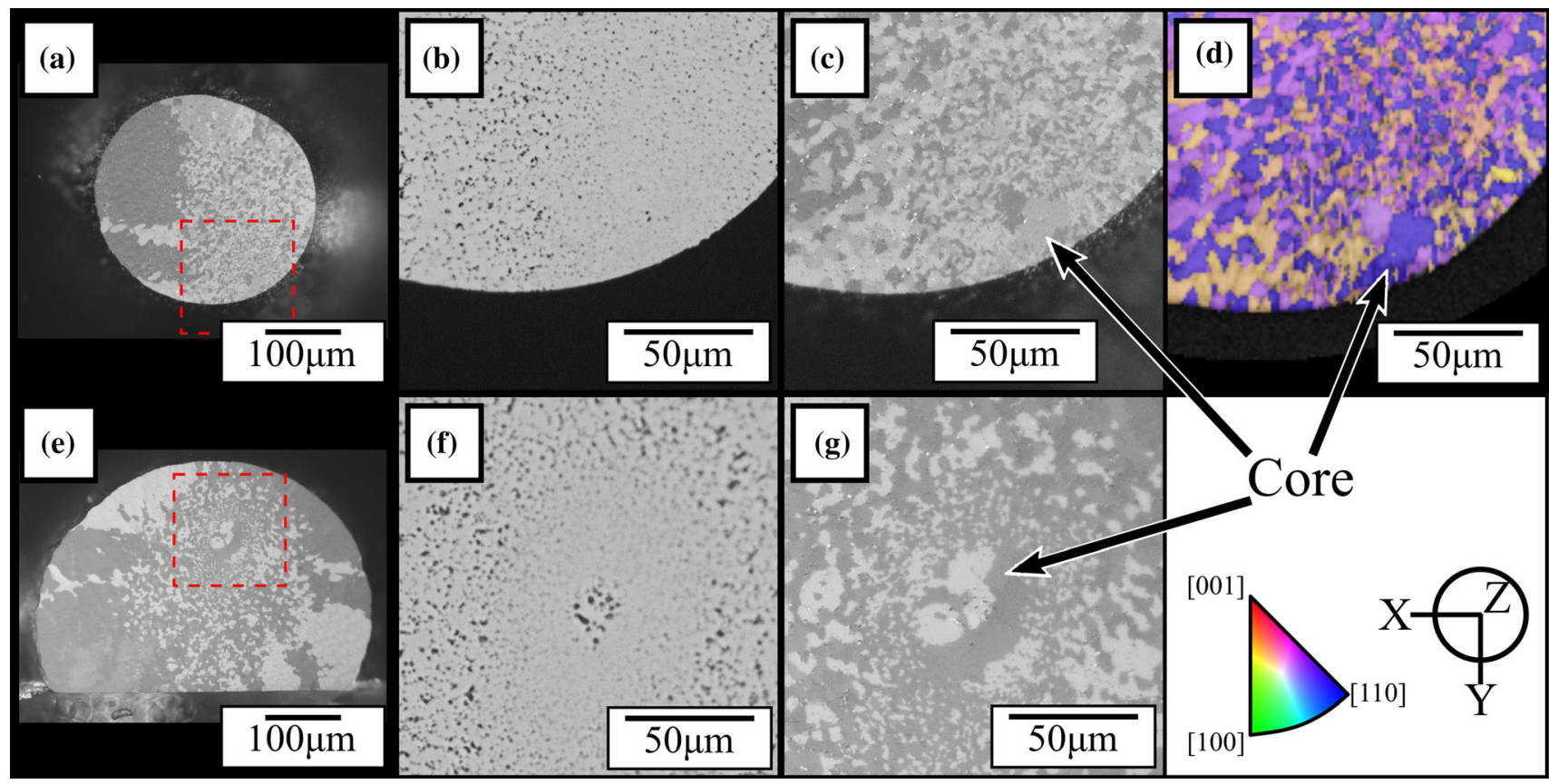

Fig. 5. Close up of 'core' structures observed: (a-d) from Fig. 3, (e-g) from Fig. 4. (b) and (c) Optical and polarised micrographs, respectively, of the region highlighted in (a); (d) EBSD-IPFY map with pattern quality, of the highlighted region from (a); ( $f$ ) and (g) optical and polarised micrographs, respectively, of the highlighted region in (e).

are twice as many twinned growth directions as for $\langle 110\rangle$, and these are closer to a continuous cone, as shown on the pole figure in Fig. 6c using $\langle 221\rangle$ as an example. For the case of $\langle 111\rangle$ growth, note that two adjacent $\beta$ Sn cyclic twins (i.e. [111] blue and [111] red in Fig. 6c) nearly coincide, with a misorientation of around $1^{\circ}$ if we treat the cyclic twin as three $60^{\circ}$ rotations. Thus, we see that cyclic twinning with a shared [100] direction and growth along $\langle 110\rangle$, or $\langle 11 w\rangle$ with $0<w \leq 1$, produces an envelope shape similar to a double cone with the nucleation point at the centre.

Considering the pole figures in Fig. $3 \mathrm{~m}$ and Fig. $4 \mathrm{~m}$ (note that for the left-hand pole figure the $z$-axis points into the page towards the left, and for the right-hand pole figure the $z$-axis points upwards), we see that the cone structure has the $\langle 110\rangle$ cyclic direction forming a ring in the direction of the cone opening (in bct such as $\beta \mathrm{Sn},\{110\}$ normals and $\langle 110\rangle$ directions are coincident vectors). Additionally, the [100] twinning direction is exactly in the centre of the $\langle 110\rangle$ ring, and corresponds well with the cone axis.

Figure 7 shows how this interlaced cone can be intersected (such as when grinding a sample) to form crescent-shaped and ring-shaped conic sections, alongside examples of real observed microstructures which match this conic section. Figure 7a shows a section forming a crescent-shaped interlaced area as exemplified in Fig. 7b-e. A conic section forming a ring Fig. $7 \mathrm{f}$ can be observed in Fig. $7 \mathrm{~g}-\mathrm{j}$. In Fig. $7 \mathrm{~d}$ and i, a primary IMC is visible in the cross-section, which is shown enlarged in the inset. In Fig. $7 \mathrm{~d}$, a primary $\mathrm{Ag}_{3} \mathrm{Sn}$ plate is visible in
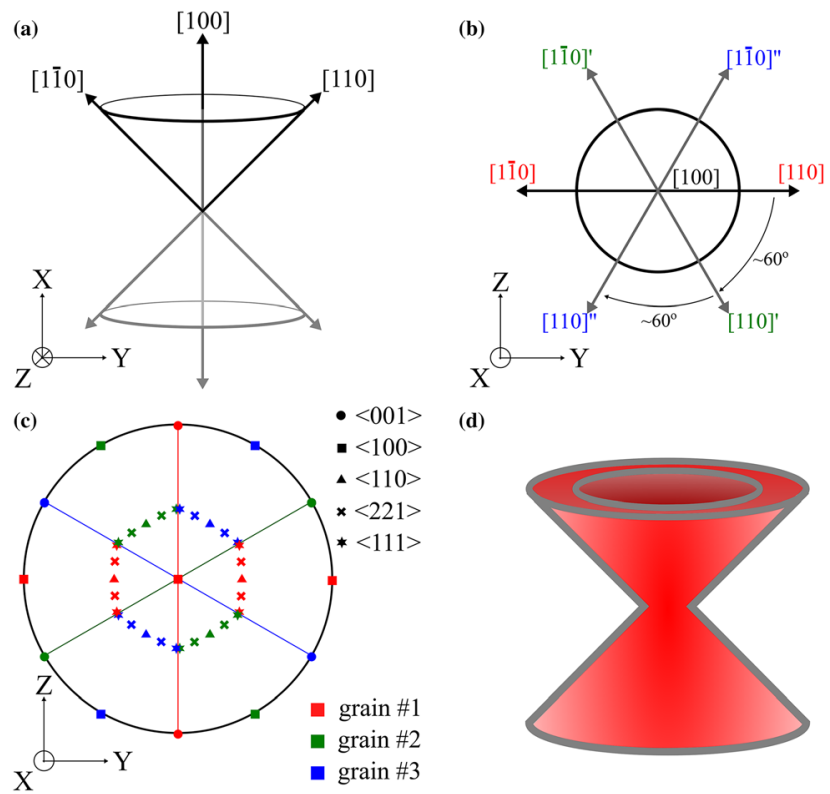

(d)

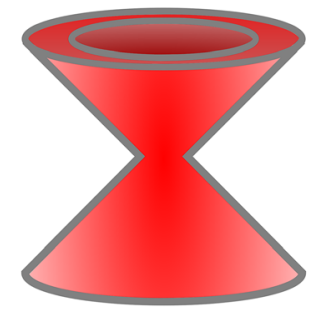

Fig. 6. Proposed conical model of interlacing. (a) The cone showing a [100] axis, with $\langle 110\rangle$ directions rotated around the [100] to form the cone; (b) [100] projection of the cone showing how $\beta$ Sn cyclic twining rotates around the cone axis forming the $\langle 110\rangle$ sides of the cone; the three cyclic twinning directions are distinguished with primes ([110], [110]' and [110]"); (c) pole figure of three cyclic twinned $\beta \mathrm{Sn}$ directions (red, green, blue), with $\langle 110\rangle,\langle 221\rangle$ and $\langle 111\rangle$ directions forming a ring (cone in 3-D) around the common [100] direction in the centre; (d) 3-D model of the hollow double cone with some thickness (Color figure online).

the optical micrograph, on the left side of the sample near the edge of the crescent-shaped interlacing, whereas in Fig. $7 \mathrm{i}$, a primary $\mathrm{Cu}_{6} \mathrm{Sn}_{5}$ rod cross- 


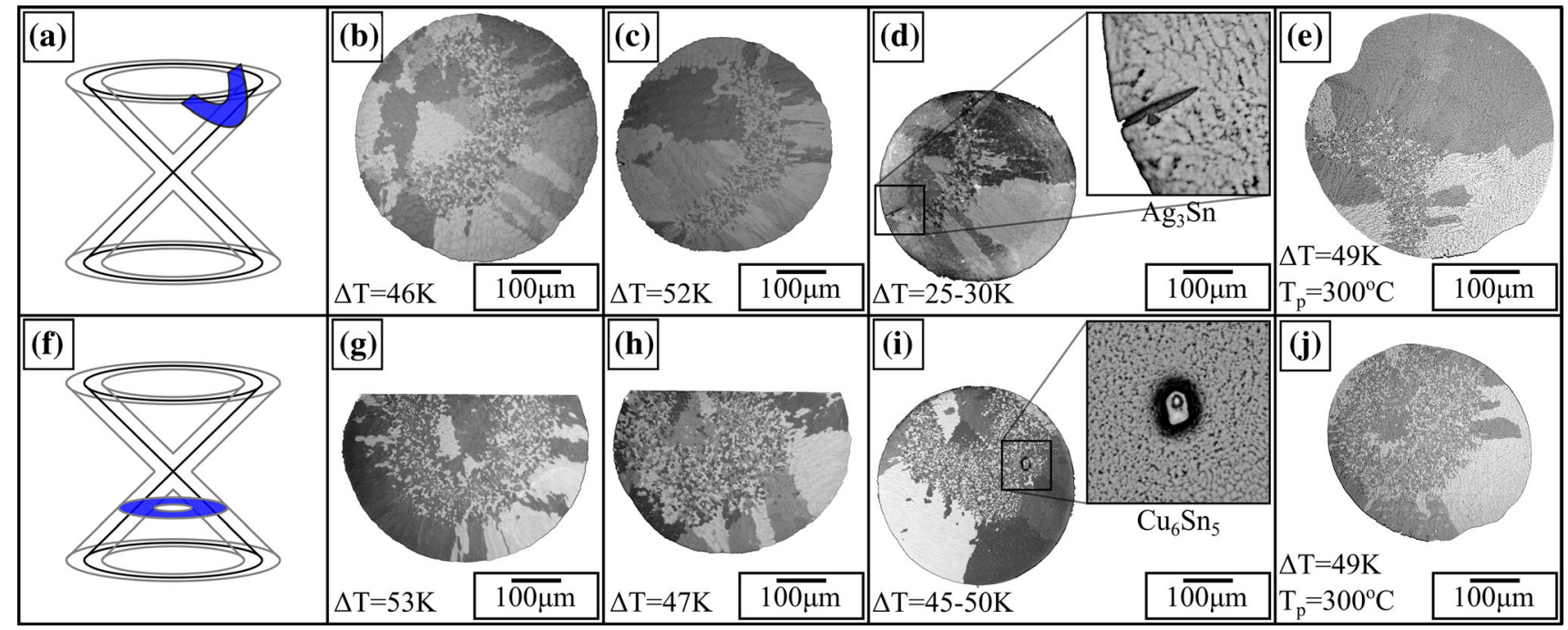

Fig. 7. (a and f) Example cross-sections through the interlacing cone, with the expected shape of interlacing in blue; (b-e) micrographs showing the crescent-shaped interlacing expected from (a); (g-j) micrographs showing a hollow ring of interlacing expected from (e); all micrographs show their nucleation undercooling $(\Delta T)$; ( $\mathrm{d}$ and I) contain an inset of the IMC particles in optical microscopy; both insets are $100 \mu \mathrm{m} \times 100 \mu \mathrm{m}$ large; (d) optical micrograph of commercial purity SAC305 sample; (I) is from SAC305 supplied by Nihon Superior. (e) and (j) are a single sample in different cross-sections, heated to a peak temperature of $300^{\circ} \mathrm{C}$ (Color figure online).

section is observed inside the crescent/semi-ring of interlacing. The cross-sections in Fig. 7e and $\mathrm{j}$ come from a single sample containing the cone morphology at different grinding depths, and show that this interlacing structure is also found after cooling from different peak temperatures.

EBSD of sections from multiple different balls provides further support for this 3-D structure. The results of some of these conic sections are shown in Fig. 8. From the $\{110\}$ pole figures and the proposed conic section, we see how the cyclic twinned $\langle 110\rangle$ directions form the cone walls, and that the $\langle 100\rangle$ twinning axis (shown as a blue triangle on the $\{110\}$ pole figures) points in the direction of the cone axis. In Fig. 8a, for example, we see that the V-shape of the interlaced region, with the centre of the cone near the sample surface, has the cone axis running left-to-right, whereas in Fig. 8b, the V-shaped interlaced region has its cone axis running up-and-down. Looking back at the reconstructions in Figs. $3 \mathrm{~m}$ and $4 \mathrm{~m}$, we see that the cone centre, or tip, seems to be near the sample surface, suggesting that this is the likely nucleation point of the cone structure. If the cone structure nucleates near the sample surface, as implied by the observed microstructures, with a random orientation, the possible growth directions are limited by the sample shape and the availability of liquid around the nucleation point. It is then probable that half of the cone structure will grow in the direction of the sample edge and terminate. The proposed nucleation and growth leads to structures observed in Fig. 8a and b, whereas if nucleation of this cone structure occurs in the bulk of the sample a full X-shaped morphology will be observed in some cross-sections. Note that, in Fig. $8 \mathrm{a}$ and b, while
Fig. 8. EBSD results of some interlacing microstructures which suggest a hollow conic growth shape. For each sample, a polarised micrograph, an EBSD IPF map and the $\{110\}$ pole figure is shown. The hollow cone structure is shown below the pole figures oriented according to it, and standardised in the fourth column. The interlaced area is highlighted in blue. The blue triangle on the $\{110\}$ pole figures show the approximate position of the $\{100\}$ twining axis of the cyclic-twinned $\beta \mathrm{Sn}$, and the hollow cone axis. (a) shows IPFZ, and $(b-e)$ are IPFX maps (Color figure online).

there are similarities in their $\mathrm{V}$-shaped area of interlacing, Fig. 8a has a near-horizontal $\langle 100\rangle$ twinning axis whereas Fig. 8b has a near-vertical $\langle 100\rangle$ twinning axis, which leads to the different shaded regions on the double cone in the final column. Figure 8c-e show how the cone structure cross-sectioned nearly perpendicularly to the cone axis results in circular, ring and crescent-shaped interlaced regions, like those reported in the literature. ${ }^{1,9,18-20}$

Figure 9 shows how the $\beta \mathrm{Sn}$ growth directions of two samples form the conical shape around the common $\langle 100\rangle$ direction. Figure $9 \mathrm{c}$ and $\mathrm{f}$ shows the 6 $\langle 110\rangle$ and the $12\langle 221\rangle$ directions forming a ring around the twinning axis/cone axis (blue triangle). Any growth direction of $\langle 11 w\rangle$ type, (with w between 0 and 1) will also lie on that ring, as shown in Fig. 6c, forming the sides of this hollow double cone.

Figure 10 shows the thickness of the interlacing regions for the samples presented in Fig. 8. The thickness of the interlacing region is seen to vary greatly in Fig. $10 \mathrm{a}$, from $73 \mu \mathrm{m}$ to $172 \mu \mathrm{m}$, depending on the position of the measurement. A similar variation of thickness is observed in the other samples in Fig. 10b-e, with all the samples showing 


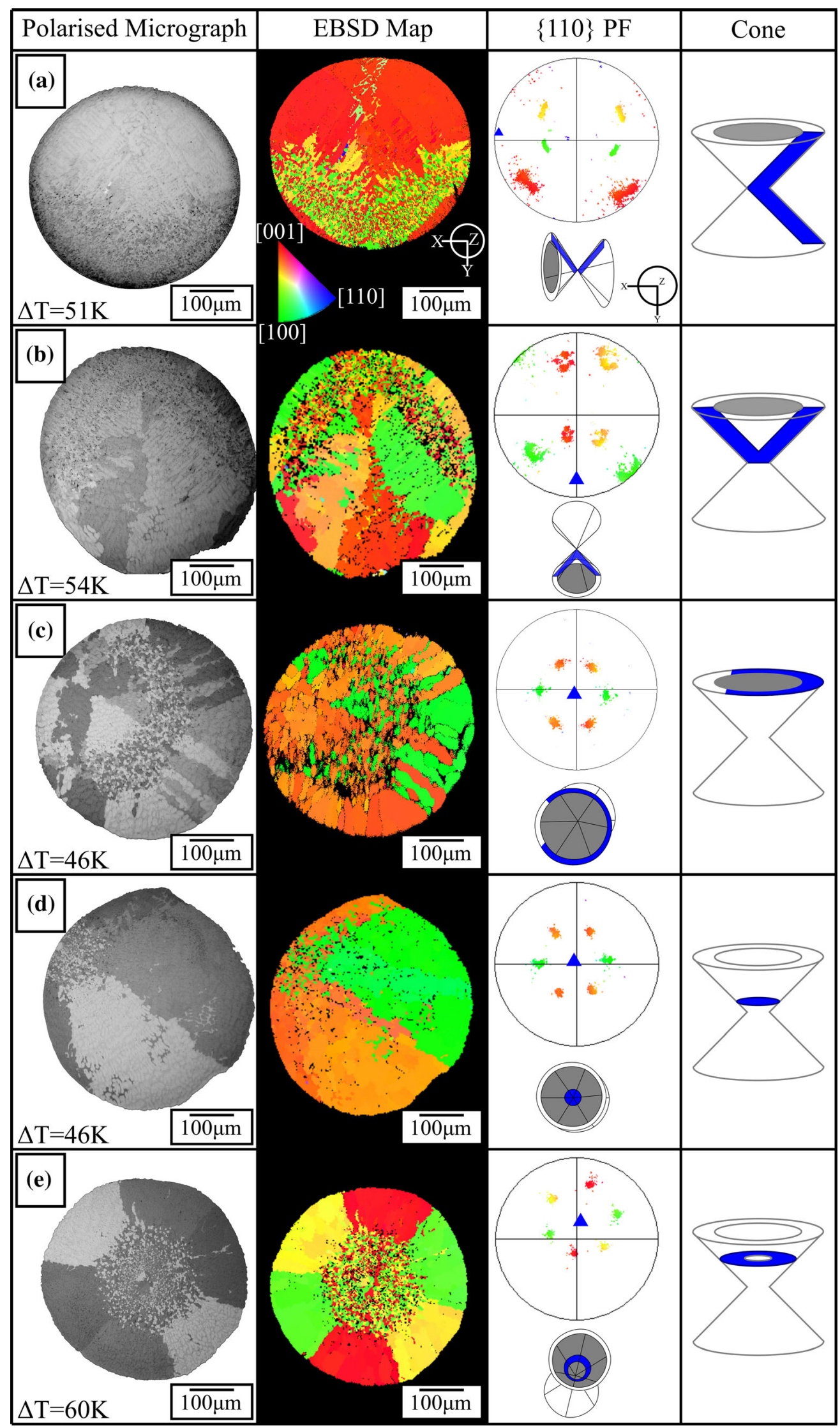




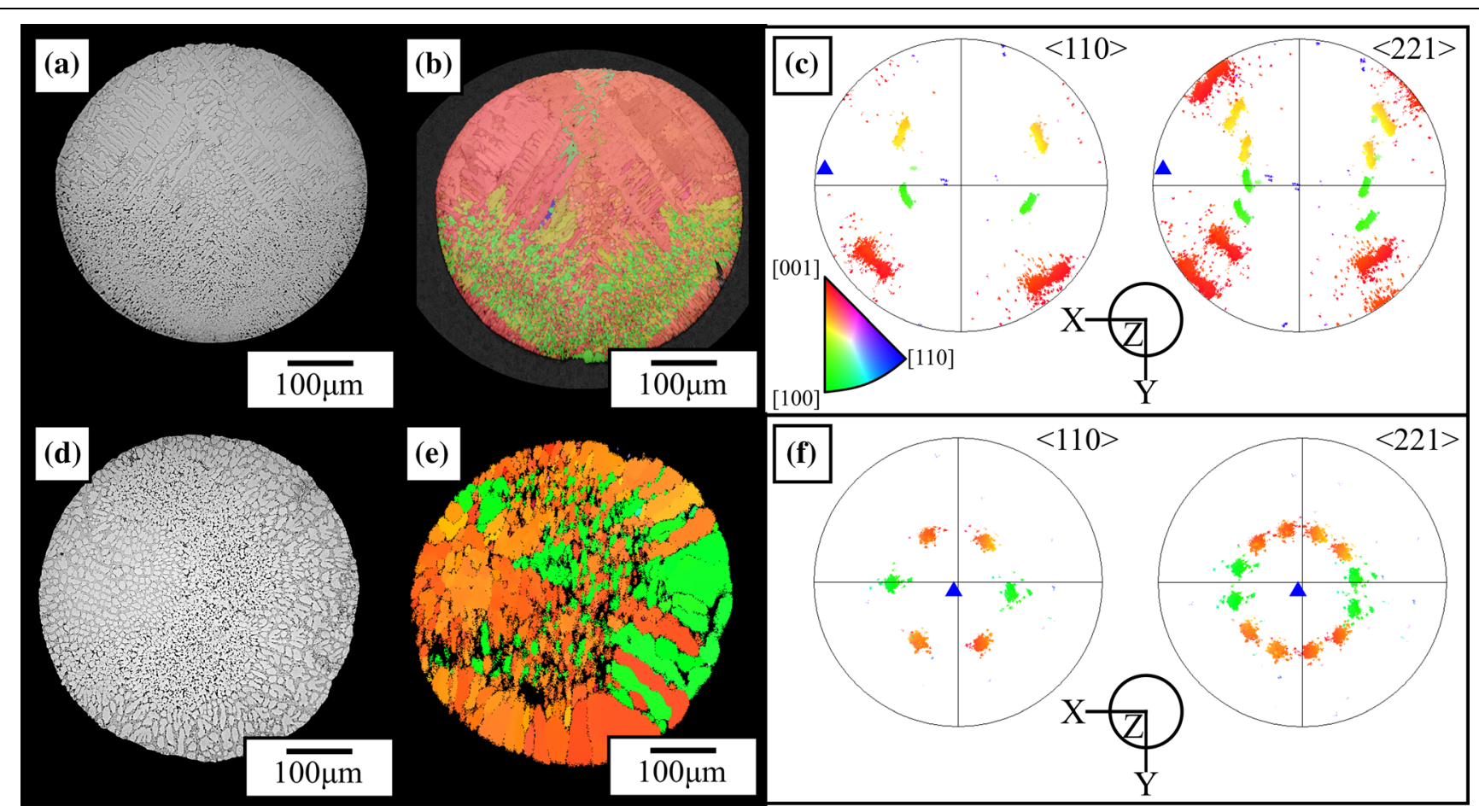

Fig. 9. $\beta$ Sn directions in two samples, from Fig. 8, showing a conic cross-section. (a) Optical micrograph, (b) EBSD IPFZ map, and (c) the $\langle 110\rangle$ and $\langle 221\rangle \beta$ Sn directions of the sample from Fig. 8a; (d) optical micrograph; (e) EBSD IPFX map; (f) the $\langle 110\rangle$ and $\langle 221\rangle \beta S n$ direction of the sample from Fig. 8c. Blue triangles in the pole figures represent the common $\langle 100\rangle$ direction of the cyclic twins and the cone axis (Color figure online).

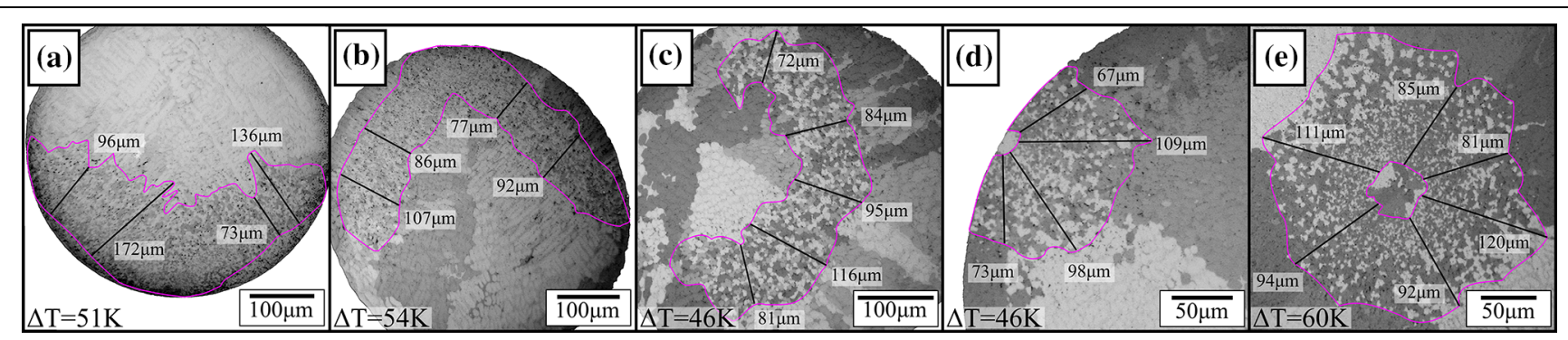

Fig. 10. Polarised micrographs of conic cross-section with the interlaced area of each sample outlined, (a-e) are samples from Fig. 8(a-e) respectively. The thickness of the interlacing is measured in selected places, as indicated.

a general range of thickness of between $\sim 70 \mu \mathrm{m}$ and $\sim 120 \mu \mathrm{m}$. Additionally, the cross-sections in Fig. 10a and $\mathrm{b}$ do not show a relationship between the thickness and the distance from the cone origin, so it is impossible to correlate the distance from origin of the samples in Fig. 10c-e where the cone axis is out of plane. Clearly, Fig. 10d is closer to the origin of the cone than Fig. 10c or e, yet the cone widths are about the same. There is some indication in the literature that thinner ring/crescent shapes exist $(\sim 12-60 \mu \mathrm{m}$, Sn-3Ag-0.7Cu $270 \mu \mathrm{m}$ solder ball, and $\sim 30 \mu \mathrm{m}$ Sn-4Ag-0.9Cu $270 \mu \mathrm{m}$ solder ball), ${ }^{19}$ indicating that ball size and composition may play a role which should be investigated further.

A further feature in this work was that samples that contained interlaced morphologies consistent with a hollow double cone (e.g. Figs. 3-8) rarely contained any observable primary $\mathrm{Ag}_{3} \mathrm{Sn}$ plates or primary $\mathrm{Cu}_{6} \mathrm{Sn}_{5}$ rods. The smallest primary $\mathrm{Ag}_{3} \mathrm{Sn}$ detected in this study was $20 \mu \mathrm{m}$ long, and between $0.8 \mu \mathrm{m}$ and $2 \mu \mathrm{m}$ thick, and smaller particles are likely to be missed in a 2-D cross section. While it is possible that larger primary IMCs were missed in samples in which only a single cross-section was studied, we note that, in Figs. 3 and 4, out of the six serial cross-sections covering most of each sample, none contained any observable primary IMCs, even though the melt undercooling reached $53 \mathrm{~K}$, which is well below the $\mathrm{Ag}_{3} \mathrm{Sn}$ and $\mathrm{Cu}_{6} \mathrm{Sn}_{5}$ metastable liquidus surfaces. ${ }^{24}$ Figure $7 \mathrm{~d}$ and $\mathrm{i}$ shows cross-sections of interlacing morphology, consistent with a hollow double-cone model, which do contain primary IMC particles. We present these examples to 
highlight that primary IMCs can be present with double-cone interlacing (i.e. the absence of primary IMCs is not a prerequisite for the hollow doublecone shape of interlacing). However, in these samples, the primary IMCs seem to be away from the likely nucleation point of the cone and are not thought to strongly influence its formation.

Additionally, from the EBSD results in Figs. 3, 4 and 8 , we see that the $\beta \mathrm{Sn}$ grain structure is consistent with a simple cyclic twinning around a common $\{100\}$ plane direction of just three $\beta \mathrm{Sn}$ grains. Even in the multiple cross-sections in Figs. 3 and 4 , only three $\beta \mathrm{Sn}$ grain orientations are observed. This result is consistent with past studies, and more complex interrelated cyclic twinning, previously reported by Ma et al., ${ }^{25}$ was not observed here in hollow double-cone morphologies. This is most likely due to the nucleation of $\beta \mathrm{Sn}$ in the solder balls before that of $\mathrm{Ag}_{3} \mathrm{Sn}$, as opposed to the droplet nucleation study of $\mathrm{Ma}$ et al., where a large $\mathrm{Ag}_{3} \mathrm{Sn}$ particle was always present as a substrate for the $\beta$ Sn.

The hollow double-cone structure identified here seems to be consistent with the cyclic twinnednucleus model by Lehman et al. ${ }^{1}$ For both their $\{101\}$ and $\{301\}$ twinned-nucleus models, extending the $\langle 110\rangle$ growth directions from such a nucleus does indeed form cone sides, as was illustrated in the pole figure in Fig. 6c. However, since Fig. 6 and the twinned-nucleus models of Lehman et al. ${ }^{1}$ all plot directions from the same type of cyclic twin, they must be geometrically consistent in this way. It is therefore difficult to confirm or rule out either twinned-nucleus model as the origin of a hollow double-cone structure.

Interlaced regions whose envelope shape is a hollow double cone seem to be common in SAC305 solder balls with a nucleation undercooling between $25 \mathrm{~K}$ and $60 \mathrm{~K}$, that are partially interlaced, and contain little or no primary IMC. From the SAC305 samples studied here, 78 of them had a partially interlaced microstructure and no primary $\mathrm{Ag}_{3} \mathrm{Sn}$ observed in the cross-section, and out of those, 11 samples could be fully confirmed to contain a hollow double-cone interlacing. Many other SAC305 samples showed 2-D microstructures consistent with a hollow double cone, but these could not be confirmed without further in-depth analysis, since this structure can only be fully confirmed in samples that were serially-sectioned with a $90^{\circ}$ rotation combined with EBSD (e.g. Figs. 3, 4), or in certain fortuitously cross-sectioned samples (e.g. Fig. 8).

The presently identified hollow double-cone shape is consistent with the frequently reported crescent and ring interlaced morphologies in 2-D sections of SAC solder balls, ${ }^{1,9,18-20}$ and also the V-shaped envelope identified in this work. We note that other interlaced morphologies are also present in micrographs in the literature, especially in samples with a higher fraction of primary $\mathrm{Ag}_{3} \mathrm{Sn}$ and $\mathrm{Cu}_{6} \mathrm{Sn}_{5}$ crystals, which are not consistent with a hollow double-cone model. Other interlaced morphologies, including those formed in the presence of primary $\mathrm{Ag}_{3} \mathrm{Sn}$, will be presented and discussed in a separate manuscript. Further work is required to deduce the 3-D shape of the envelope bounding the interlaced regions in these cases.

\section{CONCLUSIONS}

This study carried out an in-depth analysis of the shape of $\beta$ Sn interlaced regions in $\sim 500$ - $\mu$ m-diameter freestanding $\mathrm{Sn}-3 \mathrm{Ag}-0.5 \mathrm{Cu}$ solder balls. After performing serial sectioning combined with EBSD to reconstruct the 3-D volume of the interlaced regions in selected samples, and comparing with cross-sections from multiple samples, the following conclusions can be drawn:

1. Some samples have been found to contain a hollow double cone of interlacing, with the [100] twinning axis being the cone axis, and the 6 cyclic twinned $\langle 110\rangle$ growth directions, or 12 $\langle 11 w\rangle$ growth directions, forming the cone sides.

2 . The circular, ring, and crescent-shaped interlaced features reported on 2-D sections in the literature all belong to this one interlaced shape in 3-D. Additionally, a V-shaped interlaced structure was measured in this work that also belongs to the same hollow double cone structure.

3. The tip/centre of the cone is likely to be the nucleation point of $\beta \mathrm{Sn}$, and was commonly found near (and possibly at) the sample surface.

4. These cone structures were found in samples with no, or only a small amount of, primary $\mathrm{Ag}_{3} \mathrm{Sn}$ plates or $\mathrm{Cu}_{6} \mathrm{Sn}_{5}$ rods.

\section{ACKNOWLEDGMENTS}

The authors gratefully acknowledge financial support from the EPSRC doctoral training partnership grant (EP/N509486/1), and the use of Harvey Flower Microstructural Characterisation at Imperial College London. The authors would also like to express their gratitude to Dr. Sergey Belyakov for making the ingot and Yi Wang for some of the sample preparation.

\section{CONFLICT OF INTEREST}

The authors declare that they have no conflict of interest.

\section{OPEN ACCESS}

This article is licensed under a Creative Commons Attribution 4.0 International License, which permits use, sharing, adaptation, distribution and reproduction in any medium or format, as long as you give appropriate credit to the original author(s) and the source, provide a link to the Creative Commons licence, and indicate if changes were made. The 
images or other third party material in this article are included in the article's Creative Commons licence, unless indicated otherwise in a credit line to the material. If material is not included in the article's Creative Commons licence and your intended use is not permitted by statutory regulation or exceeds the permitted use, you will need to obtain permission directly from the copyright holder. To view a copy of this licence, visit http://creativecom mons.org/licenses/by/4.0/.

\section{REFERENCES}

1. L.P. Lehman, Y. Xing, T.R. Bieler, and E.J. Cotts, Acta Mater. 58, 3546 (2010).

2. V.T. Deshpande and D.B. Sirdeshmukh, Acta Crystallogr. 14,355 (1961).

3. J.W. Xian, G. Zeng, S.A. Belyakov, Q. Gu, K. Nogita, and C.M. Gourlay, Intermetallics 91, 50 (2017).

4. B.F. Dyson, J. Appl. Phys. 37, 2375 (1966).

5. Z.L. Ma, S.A. Belyakov, K. Sweatman, T. Nishimura, T. Nishimura, and C.M. Gourlay, Nat. Commun. 8, 1916 (2017).

6. Z.L. Ma and C.M. Gourlay, J. Alloys Compd. 706, 596 (2017).

7. C.M. Gourlay, S.A. Belyakov, Z.L. Ma, and J.W. Xian, JOM 67, 2383 (2015).

8. B. Arfaei, N. Kim, and E.J. Cotts, J. Electron. Mater. 41, 362 (2012).

9. L.P. Lehman, S.N. Athavale, T.Z. Fullem, A.C. Giamis, R. Kinyanjui, M. Lowenstein, K. Mather, R. Patel, D. Rae, J. Wang, Y. Xing, L. Zavalij, P. Borgesen, and E.J. Cotts, J. Electron. Mater. 33, 1429 (2004).

10. Z.L. Ma, S.A. Belyakov, and C.M. Gourlay, J. Alloys Compd. 682, 326 (2016).

11. J.W. Xian, Z.L. Ma, S.A. Belyakov, M. Ollivier, and C.M. Gourlay, Acta Mater. 123, 404 (2017).

12. M. Mueller, S. Wiese, M. Roellig, and K.J. Wolter, in Proceedings. 57th Electronic Components and Technology Conference (2007), pp. 1579-1588.
13. H. Shang, Z.L. Ma, S.A. Belyakov, and C.M. Gourlay, J. Alloys Compd. 715, 471 (2017).

14. Z.L. Ma, H. Shang, A.A. Daszki, S.A. Belyakov, and C.M. Gourlay, J. Alloys Compd. 777, 1357 (2019).

15. B. Arfaei, L. Wentlent, S. Joshi, A. Alazzam, T. Tashtoush, M. Halaweh, S. Chivukula, L. Yin, M. Meilunas, E. Cotts, and P. Borgesen, in 13th Intersociety Conference on Thermal and Thermomechanical Phenomena in Electronic Systems (IEEE, 2012), pp. 392-398.

16. B. Arfaei, S. Mahin-Shirazi, S. Joshi, M. Anselm, P. Borgesen, E. Cotts, J. Wilcox, and R. Coyle, in Proceedings of Electronic Components and Technology Conference, vol. 13748 (2013), p. 976.

17. B. Arfaei, Y. Xing, J. Woods, J. Wolcott, P. Tumne, P. Borgesen, and E. Cotts, in 2008 58th Electronic Components and Technology Conference (IEEE, 2008), pp. 459-465.

18. M. Mueller, S. Wiese, and K.-J. Wolter, in 2009 59th Electronic Components and Technology Conference (IEEE, 2009), pp. 1027-1036.

19. I. Panchenko, M. Mueller, K.-J. Wolter, E. Zschech, S. Ogawa, and P. S. Ho, in AIP Conference Proceedings (2010), pp. $245-254$.

20. M. Krause, M. Muller, M. Petzold, S. Wiese, and K.-J. Wolter, in 2008 58th Electronic Components and Technology Conference (IEEE, 2008), pp. 75-81.

21. SVG, Inkscape, and the Isometric Projection (2016), http://je roenhoek.nl/articles/svg-and-isometric-projection.html. Accessed 05 Mar 2020.

22. C.T. Rueden, J. Schindelin, M.C. Hiner, B.E. DeZonia, A.E. Walter, E.T. Arena, and K.W. Eliceiri, BMC Bioinform. 18, 529 (2017).

23. G.L.F. Powell, G.A. Colligan, V.A. Surprenant, and A. Urquhart, Metall. Trans. A 8, 971 (1977).

24. K.-W. Moon, W.J. Boettinger, U.R. Kattner, F.S. Biancaniello, and C.A. Handwerker, J. Electron. Mater. 29, 1122 (2000)

25. Z.L. Ma, J.W. Xian, S.A. Belyakov, and C.M. Gourlay, Acta Mater. 150, 281 (2018).

Publisher's Note Springer Nature remains neutral with regard to jurisdictional claims in published maps and institutional affiliations. 